\title{
RELATIONSHIP AMONG BRAIN HEMISPHERIC DOMINANGE, ATTITUDE TOWARDS LI AND L2, GENDER, AND LEARNING SUPRASEGMENTAL FEATURES
}

\author{
Mohammad Hadi Mahmoodi' \\ Sorour Zekrati ${ }^{2}$ \\ Bu-Ali Sina University, Hamedan, Iran \\ mhmahmoodi@yahoo.com'(corresponding author), s.zekrati@gmail.com² \\ First received: 22 April 2016 \\ Final proof received: 17 July 2016
}

\begin{abstract}
Oral skills are important components of language competence. To have good and acceptable listening and speaking, one must have good pronunciation, which encompasses segmental and suprasegmental features. Despite extensive studies on the role of segmental features and related issues in listening and speaking, there is paucity of research on the role of suprasegmental features in the same domain. Conducting studies which aim at shedding light on the issues related to learning suprasegmental features can help language teachers and learners in the process of teaching/learning English as a foreign language. To this end, this study was designed to investigate the relationship among brain hemispheric dominance, gender, attitudes towards L1 and L2, and learning suprasegmental features in Iranian EFL learners. First, 200 Intermediate EFL learners were selected from different English language teaching institutes in Hamedan and Isfahan, two provinces in Iran, as the sample. Prior to the main stage of the study, Oxford Placement Test (OPT) was used to homogenize the proficiency level of all the participants. Then, the participants were asked to complete the Edinburgh Handedness Questionnaire to determine their dominant hemisphere. They were also required to answer two questionnaires regarding their attitudes towards L1 and L2. Finally, the participants took suprasegmental features test. The results of the independent samples t-tests indicated left-brained language learners' superiority in observing and learning suprasegmental features. It was also found that females are better than males in producing suprasegmental features. Furthermore, the results of Pearson Product Moment Correlations indicated that there is significant relationship between attitude towards L2 and learning suprasegmental features. However, no significant relationship was found between attitude towards L1 and learning English suprasegmental features. The findings of this study can provide English learners, teachers and developers of instructional materials with some theoretical and pedagogical implications which are discussed in the paper.
\end{abstract}

Keywords: Suprasegmental Features, Hemispheric Dominance, Attitude towards L1, Attitude towards L2

One of the main aims of the language teaching methods is to enable the learners to communicate primarily orally with other people in different contexts, without facing serious problems. To gain this goal, learners should be taught how to speak intelligibly and listen meticulously, and this just happens when they have mastery over pronunciation elements such as segmental and suprasegmental features. In fact, teaching pronunciation for the sake of listening and speaking in second/foreign language has been the focus of different methods such as Audiolingual and Situational language teaching for many years (Rogerson-Revell \& Miller, 1994), and is still considered as a factor which determines the speakers' failure or success in terms of conveying intended meaning (Pachler \& Field, 1997). In addition to its outstanding role in conveying meaning, pronunciation, helps speakers with "a sense of identity", and contributes to "signal group membership" (Jenkins, 2004).
Suprasegmental or prosodic features which stand in contrast to segmental features, i.e. vowels and consonants, are phonological units (Chun, 2002) which not only accompany single sounds but also extend over syllables, words, or longer units of speech such as phrases and sentences(Ladefoged, 2010). Such suprasegmental features as stress, tone, and intonation play crucial roles in communication, to the extent that any interference and error in using them can "inhibit the transmission of meaning itself; negating or contradicting the intention of the speaker" (Nash, 1971, p. 138). Thus, believing in suprasegmental features' outstanding role in communication, many scholars have discussed the ways in which they can be taught and learnt more effectively in classrooms(e.g. Brazil, Coulthard, \& Johns, 1980; Fotovatnia \& Omidi, 2013; Ladefoged, 2010; Roach, 2002). The results of these studies have shown direct relationship between suprasegmental features learning and different variables such as attitudes towards L1 and L2. The 
main problem with these studies is not only their unidimensionality, i.e. considering one aspect of suprasegmental features and discussing that in relation to one independent variable, but also their controversial results and implications which could not solve the problem of misusing these features in daily conversations at least in Iranian Context, where many EFL learners have problem with word stress, assimilation (a process through which one sound becomes more like a nearby sound within a word or between words) and intonation. In fact, intonation conveys attitudes, emotions, and even grammatical points much faster than the structure of the sentence (Roach, 2002). Thus, not being able to comprehend or use intonation patterns properly hinders communication. The same thing happens when the stress of words are misplaced and mispronounced. In addition, non-native language learners may face challenges when listening to native speakers who speak with lots of reductions and assimilations, if they do not know these features well. As an example, due to being unfamiliar with reduction forms, a non-native language learner may face problem when hearing a native speaker saying "ya couldn' a done it" or "dj' ask'er" which are reduced form of "you could not have done it" and "did you ask her" respectively. Many factors can hinder the process through which non-natives perceive, comprehend, or produce suprasegmental features. That is why this study, in an attempt to fill in the previously mentioned gaps, and to add research knowledge on the process of suprasegmental features learning, examines the relationship among suprasegmental features learning, and biological and non-biological factors such as hemispheric dominance, and attitude towardsL1 and L2.

\section{Review of literature}

The discussion on the role of brain hemispheres in controlling each side of the body and performing various functions started decades ago in the 1860 s, when Broca found out that the frontal lobe of the left hemisphere serves a vital role in the production of speech (Broca, 1861). Shortly after Broca, Wernicke in 1874 began pursuing his own research into the domain of brain diseases and found out that some of the language comprehension deficits were the result of damage to the left posterior, superior temporalgyrus, which is now referred to as Wernicke's area. Following the works of these two pioneers, and with outstanding advances in imaging techniques, particularly Magnetic Resonance Imaging (MRI) and Positron Emission Tomography (PET), other scientists could image subcortical brain structures, and consequently found many other body functions related to specific parts of the brain. The results of these studies indicated the right brain superiority in visuo-spatial abilities (Goldstein, Joynt, \& Hartley, 1975; Corkin, 1978; Davidoff,
1982, Ratcliff,1982; McKeever \& Jackson, 1979), face perception (Milner, 1968; Yin,1970 ), musical skills (Goodglass \& Calderon,1977), sensory and motor attention and memory (Devinsky \& D'esposito, 2004); and left hemisphere advantage in serial order perceptions, verbal cognition, motor responses, and logical, sequential analysis (Geschwind, 1975).

Evidence supporting right hemisphere dominance Among these functions, language learning has been given attention to a great extent and has been studied by many scholars who were intended to find the role of hemispheric dominance in first or second language learning. These studies have scrutinized the role of hemispheres in learning different skills and sub-skills and even specific features of language such as prosodic ones. As an example, Gandour et al., (2004) investigated the roles of hemispheres in the perception of prosodic language. Their proposed model of speech prosody perception showed that right hemisphere regions are primarily involved in complex-sound analysis such as intonation, while left hemisphere regions are activated whenever language processing requires more than the auditory analysis of the complex sound.

Anaki, Faust, and Kravetz (1998) investigated the relationship between semantic priming (the idea that we process stimuli better if it is preceded by a semantically related prime compared to an unrelated prime; for example, response to " hospital" is faster when preceded by "doctor" than when it is preceded by "bread") and hemispheric dominance. The results of their investigations showed the significance of right hemisphere in metaphoric comprehension. Arzouan, Goldstein, and Faust (2007), in a similar study, demonstrated that right hemisphere mechanisms play significant role in understanding metaphoric expressions. However, they believed that brain hemispheres work together in a complex dynamical pattern while figurative and literal comprehension take place. Accumulated evidence from acoustical studies, neuro-imaging, neuropsychology, and neuro-anatomy suggests right hemisphere activation during suprasegmental features processing in adults and children (Baum \& Pell, 1999; Friederici \& Alter, 2004; Gandour, et al., 2004; Jusczyk, 1997; Shipley-Brown, Dingwall, Berlin, Yeni-Komshian, \& Gordon-Salant, 1988)

Tucker, Watson, and Heilman (1977), as major proponents of the view that right hemisphere controls prosody, conducted a research with 16 participants including eight right hemispheredamaged patients and eight normal control subjects and concluded that the right hemisphere was involved in the production of affective prosody. In addition to affective prosody, numerous studies have examined hemispheric dominance and the production of linguistic prosody. Among the types of evidence that have been brought to bear on this 
issue are results of studies on participants with right and left hemisphere damages done by Weintraub, Mesulam \& Kramer (1981) in which the right hemisphere's potential role in repetition, discrimination, and spontaneous production of linguistic prosody was examined. $\mathrm{He}$ used declarative and interrogative sentences besides sentences with emphatic stress as stimuli to test 19 participants, including participants with right brain damage and control participants without brain injuries. The obtained results of the study lend support to right-hemisphere dominancy for prosodic production since the subjects with the right hemisphere damage were not as good as the subjects of the control group in distinguishing prosodic features with emphatic or phonemic information. In a similar study, Blumstein and Cooper (1974) designed an experiment to explore the nature of intonation processing in hemispheres. Thereupon, they conducted two dichotic experiments with filtered intonation contour in the first and nonfiltered intonation in the second one. The results suggested that while both hemispheres are involved in normal language perception, apprehension of intonation contours is dedicated to the right side. The obtained results of this study were confirmed in another experiment carried out by Shipley-Brown et al. (1988) in which, using the dichotic listening, paradigm laterality for affective and linguistic prosody was examined.

Evidence supporting left hemisphere dominance Despite the above mentioned findings, which upheld the view that understanding suprasegmental or prosodic features takes place in the right hemisphere, converging evidence from neuroimagining (Klein, Zatorre, Milner, \& Zhao, 2001), dichotic listening (Lancker \& Fromkin, 1973; Moen, 1993; Wang, Jongman, \& Sereno, 2001), and lesion deficit (Eng, Obler, Harris, \& Abramson, 1996; Gandour \& Dardarananda, 1983; Yiu \& Fok, 1995) confirm left hemisphere involvement in the perception of emotional prosody at the syllable or word-level structures. Other studies also confirmed that left hemisphere has the main role in processing auditory inputs (Meyer \& Yates, 1955), recognition of linguistic materials, such as digits (Broadbent \& Gregory, 1964; Bryden, 1963; Kimura, 2000), and vowel syllables (Shankweiler \& Studdert-Kennedy, 1967).

In a lesion study, Schirmer, Alter, Kotz, and Friederici, (2001) examined brain lateralization of prosodic language. 11 right and left -hemispherelesion patients were asked to recognize prosodic feature of two different syntactic structures. The equivocal results of acoustical analyses on F0 (fundamental frequency) and time structure indicated the left-hemisphere superiority for the processing of linguistic prosody. The superiority of the left hemisphere was also reported in processing metaphoric sentences (Rapp, Leube, Erb, Grodd, \& Kircher, 2004; Stringaris, Medford, Giampetro, Brammer, \& David, 2007), tone (Moen, 1993; Wang et al., 2001) and pitch when considered linguistically (Lancker \& Fromkin, 1973).

\section{Gender and learning suprasegmental features}

Recent research has shown that the subtle differences, which exist in the way the females' and males' brains process language, cognition, information, emotion, etc. (Sabbatini, 2000), are basically due to the structure of their brain (Harasty, Double, Halliday, Kril, \& McRitchie, 1997; Shaywitz, et al., 1994). As Harasty et al., (1997) claim: "females have proportionally larger Wernicke and Broca Language-Associated Cortical Regions" and have stronger connections across the left and right hemispheres, this is while men have stronger connections between the front and back regions which makes the parietal region more important for them (Shaywitz, et al., 1995). Furthermore, neurological studies have proved that while women are able to use the posterior temporal lobes simultaneously during linguistic processing, men just use one hemisphere at a time, especially the left one while developing language structures or vocabularies (Kansaku, Yamaura, \& Kitazawa; 2000). Scholars have also found that as females tend to use areas of the brain that are devoted to emotional and verbal functioning, males use areas related to mechanical and spatial tasks (Moir \& Jessel, 1989). Regarding phonological tasks, Shaywitz et al. (1995) provided clear evidence for sex differences in the brain functional organization for language learning, and indicated that while brain activation in females engages more diffuse neural systems which involves both right and left inferior frontal gyrus regions, in males the pattern is completely different, and the activation is lateralized to the left inferior frontal gyrus.

In conclusion, based on the different psycholinguistic, psychological, and neurological studies, which have scrutinized both hemispheres and their lateralized functions; it seems that prosodic features, mainly based on their functions, can be processed in both hemispheres.

\section{Attitudes towards L1/L2 and learning suprasegmental features}

Besides hemispheric dominance, many scholars have devoted time to investigate the effect of positive or negative attitude on learning process. These studies got much more attention after Jones (1950) who found significant positive relationship between attitudes and learning Welsh language.

In one of the earliest studies on the role of attitude in second language acquisition, Gardner (1976) gathered three different samples consisting of Anglophones studying French as a second language in a monolingual milieu, Anglophones 
studying French in a bilingual setting, and Francophone students studying English in a French/ English milieu. The relationships among ten predictor variables and five variables representing possible linguistic and non-linguistic outcomes of second language programs were calculated and it was concluded that in both bilingual and monolingual milieu, although it is motivation which directly affects students achievement, attitudes serve to influence appreciably the students' motivational level and thus is, albeit indirectly, involved in mediating students achievement in a second language.

In the same year, Suter (1976) suggested that students, who are more concerned about their pronunciation, perform much better in pronouncing target language. As Cenoz and Garcia Lecumberri (1999) pointed out, in many cases, speakers tend to use the pronunciation they value as more positive, or try to choose the pronunciation with which they are identified for both their first and second languages. This point is related to the fact that attitude can be both culturally directed and language directed. In fact learning intonation, accent, and pronunciation of the second language is to a great extent affected by learners' sense of identity and attitude towards second culture and language. As many studies have pointed out, foreign language learners who have positive attitude towards second culture and language, tend to integrate with the target society and therefore adopt the favorite accent (Cakir \& Baytar 2014; Darabad, 2003; Moyer, 2007; Pourhosein Gilakjani 2012; Pullen, 2011; Sparks \& Ganschow, 1991).

Elliot (1995) conducted a dedicated study on the relationship between L2 attitude and foreign language pronunciation learning using the Pronunciation Attitude Inventory (PAI). The results of correlation between students' performance on pronunciation test and their attitude towards L2indicated that positive L2 attitude was the main variable in relation to Spanish language pronunciation, and that students who were more concerned about their pronunciation, had more positive attitude towards target language and; therefore, were more successful in imitating correct pronunciation.

Zhang and Yin (2009) analyzed some frequently occurring problems in learning English pronunciation and concluded that attitude towards the target language and its culture can affect achievement in pronunciation. They argued that L2 attitude and motivation can support language learning and either accelerate or hinder pronunciation skills development.

Moyer (2007) in a dedicated study on accent, attempted to find out whether language attitudes are relevant to phonological attainment and also whether there are apparent differences between attitudes towards the target language, and attitudes towards the target culture when learning accent or not. To this end, the researcher asked some university students to complete an open-ended and scalar questionnaire on their language background and socio-psychological orientation as concerned English and the USA. The obtained results of different analytical procedures indicated that 1) Learners with minimal confidence in their language abilities were judged to have more foreign accent 2) Learners who perceived themselves as highly fluent obtained higher accent rate. Based on the findings three main conclusions were stated: (1) Language attitudes, as well as age and the length of residence in the target language environment, are significant factors for acquiring native like accent, (2) Although attitudes towards the target language and towards target culture are all significant, it seems that attitudes towards the target language itself are more strongly linked to accent, and (3) Both experience and a positive orientation are important for attaining authenticity in accent. Taking into account the effect of different variables on the perception and production of suprasegmental features, this study set out to examine relationship among hemispheric dominance, attitude towards L1 and L2, gender, and learning suprasegmental features

\section{Purpose of the study and research questions}

To fill in previously mentioned gaps and to examine the relationship among the above-mentioned variables, we tried to find out if any significant difference existed between right-brained and leftbrained EFL learners regarding their mastery of suprasegmental features. Then, attempts were made to find any significant difference between male and female EFL learners in learning suprasegmental features. Following these two issues the relationships between EFL learners' attitude towards their L1 and L2, and learning English suprasegmental features were investigated.

\section{METHOD \\ Participants}

The participants of this study were 200 (intermediate) EFL learners from private English language teaching institutes in Esfahan and Hamedan provinces, Iran. Care was taken not to include any participants whose mother tongue was something other than Persian in order to omit the effect of any extraneous variable. The participants were chosen from the available intermediate classes in the institutes through convenience sampling procedure over five semesters during 2014-2015. Participants were 74 male and 126 female learners who met up two times per week, and received nearly 32 hours of language training per semester. Their age range was between 15 and 35 . 
Instruments and Materials

Edinburgh Handedness Inventory (EHI)

The Edinburgh Handedness Inventory was developed and validated in 1971 by R.C. Oldfield as a measurement scale to assess the hemispheric dominance of a person. It contains ten questions on a 5-point scale with the reported reliabilities of 0.940 to 0.980 (Ransil \& Schachter, 1994), and ranges from -100 for strong left-handedness to +100 for strong right-handedness.

Attitude towards English Language Questionnaire Attitude towards English Language Questionnaire, which was developed by Zainol Abidin, Pour Mohammadi, and Alzwari (2012), was used in this study in order to measure the participants' attitudes towards English. This 45-item questionnaire was validated through expert view and its reliability was estimated through Cronbach's Alpha $(r=.87)$ for this study.

\section{Attitude towards Persian Language Questionnaire}

The 124-item questionnaire of attitude towards Persian language which was designed and developed by Davari Ardekani and Jalilian (2013) was adapted in this study. Among all the available items and based on the factor analysis, only 25 directly attitude related items were chosen and adopted for this study. The adapted questionnaire was piloted on 30 participants and its reliability, as calculated using Cronbach's Alpha, was found to be .85 . The 25 -item questionnaire was also validated through expert view, pilot study and factor analysis.

\section{Speech Analyzer Software}

Speech analyzer software is a full-featured acoustic analyzer for professional comparative studies, and language learning activities. In this study it was used to play the original audio file, and show the accurate visual representation of the pitch and the intensity of the speech or sentences, words, and phonemes which were recorded for each participants. The possibility of viewing all patterns in one single screen, and the overlay option of the software made it possible for the researcher to perform comparative analysis.

\section{Oxford Placement Test}

In order to reassure that the participants are homogeneous in terms of language proficiency level, a version of Oxford Placement Test called Solutions Placement Test (Edwards, 2007) was used in this study. This test contains 50 multiple-choice questions of grammar, vocabulary and reading and 10 graded comprehension questions to assess the students' language knowledge from Elementary to Intermediate level.

\section{Suprasegmentals Learning Test (SLT)}

In order to assess the suprasegmental features knowledge of the participants, a 55-item list of suprasegmental features containing 15 sentences with different functions, 20 items related to assimilation and 20 items related to word stress was designed based on the provided samples in Headway series books (Soars \& Soars, 2009). The items were directly collected from Headway series for the sake of validity and the list of the items was modified and validated through expert view. The reliability of the Suprasegmentals Learning Test and its sub-sections, after being piloted, was computed using Cronbach's Alpha formula. The reported reliability for the Suprasegmentals Learning Test (SLT) was reported to be .70 .

The reliability of each section of SLT was computed separately. The results are shown in Table 1.

Table1. Cronbach's Alpha Reliability Statistics for Sections of SLT

\begin{tabular}{cccc}
\hline & Cronbach's Alpha & Cronbach's Alpha Based on Standardized & N of Items \\
\hline Intonation & .869 & .872 & 15 \\
Word Stress & .802 & .798 & 20 \\
Assimilation & .772 & .765 & 20 \\
\hline
\end{tabular}

As it is shown in Table1, the reliability index of each section was high enough to confirm the reliability of the test to be used in this study.

\section{Data collection procedure}

To start, the Oxford Placement Test was given to the selected language learners in order to make sure that they were truly intermediate level students. The results of the test, which were interpreted based on the OPT classification, showed that all the participants except 16 learners were at the right level. Thus, the sampling procedure was continued to replace identified language learners with intermediate level students. Then, the Edinburgh
Handedness Inventory (EHI), the Attitude towards English Questionnaire, and the Attitude towards Persian Language Questionnaire were given to the participants in three different sessions.

Then, using laptops and headphones, each subject listened to each of the 15 sample sentences once and then repeated the sentences and recorded his/her voice by the voice recorder. The researchers cut the recorded audios using online software and changed the format of the recorded audio to Wave in order to make them readable for the Speech Analyzer software. The data collection procedure for word stress and assimilation parts was the same. 
Next, using Speech Analyzer software the recorded audios were overlapped to the original ones and based on the amount of similarities between the two patterns, a score among 1 (for totally wrong patterns), 2 (for moderately similar but correct patterns), and 3 (for highly similar and correct patterns) was given to each sentence. In this way, each person had a total score out of 45 for intonation patterns.

For word stress and assimilation items, each person could get 1 for wrong answer and 2 for correct answer. The total score for each part could be 40 for each testee. Two raters rated each person on all suprasegmental features items and the average of their scores was considered as every participant's final score. At the end, a total score out of 125 was obtained for each person. The inter-rated reliability was computed in order to make sure that the scores were reliable $(r=.76)$.

\section{RESULTS AND DISCUSSION \\ Research Question 1: Testing the differences between right-brained and left-brained EFL learners regarding their mastery of suprasegmental features.}

To answer the first question, all the participants, regardless of their gender and based on the results of Edinburgh Handedness Inventory (EHI), were divided into two groups of right-brained and leftbrained learners. Then, an independent sample Ttest was run to test any probable differences between the right-brained and the left-brained participants regarding learning and applying suprasegmental features. However, before presenting the main results, the descriptive statistics are provided below in Table 2 .

Table 2. Descriptive statistics for SLT scores of right and left brained participant

\begin{tabular}{ccccc}
\hline \multicolumn{2}{c}{ Groups } & N & Mean & Std. Deviation \\
\hline \multirow{2}{*}{ SLT } & Left-Brained & 159 & 98.79 & 9.36 \\
& Right-Brained & 41 & 93.92 & 8.47 \\
\hline
\end{tabular}

As displayed in Table 2 the left-brained participants $(\mathrm{M}=98.79)$, outperformed the rightbrained ones $(M=93.92)$ in SLT.

To find out whether there was any significant difference between the right-brained and leftbrained participants regarding suprasegmental features learning; an independent sample t-test was run.

Table 3. Independent sample t-test for SLT scores of the right-brained and left-brained participants

\begin{tabular}{llccccc}
\hline & & \multicolumn{3}{c}{$\begin{array}{l}\text { Levene's Test for Equality } \\
\text { of Variances }\end{array}$} & \multicolumn{3}{c}{ t-test for Equality of Means } \\
\hline \multirow{3}{*}{ SLT } & F & Sig. & t & df & Sig. (2-tailed) \\
\hline & $\begin{array}{l}\text { Equal variances } \\
\text { assumed }\end{array}$ & .87 & .35 & 3.02 & 198 & .003 \\
$\begin{array}{l}\text { Equal variances not } \\
\text { assumed }\end{array}$ & & & 3.21 & 67.42 & .002 \\
\hline
\end{tabular}

As Table 3 indicates, the first two columns are related to the Levene's test which is used to check the assumption of homogeneity of variances. In this case, the variances were assumed to be equal since the Levens' test was not significant ( $p>.05)$, and the assumption was not violated. The third column presents the obtained results of an independent ttest. As it can be seen in Table 3, a statistically significant difference was found between the rightbrained and the left-brained participants in their performance on SLT $(\mathrm{t}(198)=3.02, \mathrm{p}<.05)$. Thus, it can be concluded that the left-brained participants $(\mathrm{M}=98.79, \mathrm{SD}=9.36)$ significantly outperformed the right-brained ones $(\mathrm{M}=93.92, \mathrm{SD}=8.47)$ on SLT. Thus, rejecting the first null hypothesis, we concluded that there existed a significant difference between the left-brained and right-brained participants with superiority of left-brained ones over the right-brained participants regarding their mastery of suprasegmental features.
These results are in line with previous research findings which confirmed the supremacy of left hemisphere over right hemisphere in learning prosodic language (Lancker \& Fromkin, 1973; Meyer \& Yates, 1955; Moen, 1993; Wang et al., 2001). The results are also compatible with the findings of a more recent study conducted by Schirmer et al., (2001) which lent support to the involvement of the left hemisphere regions in processing linguistic prosody. Moreover, the finding is in agreement with that of a study done by Klein et al., (2001) in which the left hemisphere was proved to be dominant for processing pitch in a linguistic context.

The findings are supporting "dual pathway" model (Friederici \& Alter, 2004) which states that laterality of processing, to a great extent, is dependent on the function of the stimulus' content. Therefore, the more linguistic the acoustic signal and its content, the more left hemisphere regions are 
engaged (Schirmer et al., 2001; Klein et al., 2001). Consequently, such prosodic information as intonation in English which has linguistic function too, is dominantly processed in the left-hemisphere language-related regions.

In a similar vein, the functionalist hypothesis states that the function of a stimulus, and not physical stimulus itself, determines the hemisphere which is going to process it (Zurif, 1974). In other words, the extent to which suprasegmental (prosodic) features are enclosed to segments is deemed an important factor. Hence, such features as intonation and stress, which can carry linguistic meanings, are processed in the dominant, left hemisphere. Stress, which involves the relations between adjacent syllables, appears to be both segmental and non-segmental and still is assumed to be processed in the left hemisphere (Behrens, 1989).

Based on the above mentioned evidences, and the fact that one of the main role of intonation is minimally distinguishing the function of different sentence as in "she has eaten the cake" and "she has eaten the cake?" we can conclude that intonation is also processed in the left dominant hemisphere. In other words, if intonation patterns have linguistic function (Roach, 2002), and if linguistic patterns, which need to be processed beyond the auditory analysis of the complex sound, are analyzed and processed in task dependent regions in left hemisphere (Roach, 2002), then we can conclude that this kind of speech prosody, called intonation with discourse or grammatical functions, is processed in the left hemisphere.

Putting aside the effect of function on laterality of processing, we may elaborate on the role of temporal transition, size and domain. According to Jäncke, Wüstenberg, Scheich, \& Heinze (2002), while left hemisphere regions process faster temporal transitions such as segmental cues, and linguistic prosodic cues, right hemisphere regions are more sensitive to emotional language stimuli and slower temporal transitions. Accordingly, intonational patterns with linguistic function will be processed in the left, and emotional and attitudinal functions will be analyzed in the right hemisphere.

Word stress and assimilation are assumed to be processed in the left hemisphere too, due to the fact that 1) they are language related issues and almost all language related factors and skills are processed in the left hemisphere (Mateer, 1983), and that 2) they both follow certain kinds of rules and logics that the brain automatically analyzes when processing them. Thus, since left hemisphere is responsible for processing rules, we can conclude that assimilation and word stress are processed in the left regions.

Laboratory studies done by Behrens (1989) and Wang et al., (2001) have also shown that stress contrasts, e.g. 'hot dog' or 'hotdog', are perceived and produced poorly by left hemisphere lesion patients (Behrens 1989; Wang et al., 2001). Baum and Pell (1999) also reported that patients with the left hemisphere damage had problem in identifying word stress and making judgment based on linguistic stress, which in turn indicated left hemisphere engagement in recognizing and producing word stress.

It seems apparent from the findings presented in this study; as well as from the investigations of aphasic language, neurological, acoustical, neuroimaging, neuropsychology, and neuro-anatomy studies (Baum \& Pell, 1999; Moen, 1993; Meyer \& Yates, 1955; Wang et al., 2001) that the left hemisphere is to a great extent involved in processing suprasegmental or prosodic features. In particular, perception and processing of normal language may involve both hemispheres simultaneously, with the left hemisphere being responsible for such suprasegmental features as intonation with linguistic and discourse functions, word stress and assimilation; and the right hemisphere carrying out the analysis of other suprasegmental features such as pitch and intonational patterns with attitudinal and emotional functions.

Research Question 2: Testing the differences between males and females regarding their mastery of suprasegmental features.

In order to answer the second question and find any probable difference between males and females regarding their mastery of suprasegmental features, the participants were divided into two groups based on their gender, and then an independent samples ttest was run. The descriptive statistics for gender differences regarding suprasegmental features scores are presented in Table 4.

Table 4. Descriptive statistics for SLT scores for males and females

\begin{tabular}{lcrcc}
\hline & Groups & $\mathrm{N}$ & Mean & Std. Deviation \\
\hline \multirow{2}{*}{ SLT } & females & 126 & 99.82 & 8.95 \\
& males & 74 & 94.32 & 9.11 \\
\hline
\end{tabular}

As can be seen in Table 4, female participants $(M=99.82)$ outperformed the male participants $(M=$ 94.32) regarding their mastery of suprasegmental features. In order to check for any statistically significant differences between the two genders, an independent sample t-test was run. The results are displayed in Table 5. 
Table 5. Independent sample t-test for SLT scores of male and female participants

\begin{tabular}{llccccc}
\hline & & \multicolumn{2}{c}{$\begin{array}{l}\text { Levene's Test for Equality } \\
\text { of Variances }\end{array}$} & \multicolumn{3}{c}{ t-test for Equality of Means } \\
\hline & F & Sig. & t & df & Sig. (2-tailed) \\
\hline SLT & .509 & .477 & 4.16 & 198 & .00 \\
\hline $\begin{array}{l}\text { Equal variances } \\
\text { assumed }\end{array}$ & $\begin{array}{l}\text { Equal variances not } \\
\text { assumed }\end{array}$ & & & 4.14 & 154.8 & .00 \\
\hline
\end{tabular}

As it is presented in Table 5, the assumption of homogeneity of variances was met and a statistically significant difference was found between male and female participants on SLT $(\mathrm{t}(198)=4.16, \mathrm{p}=.47)$. Females $(\mathrm{M}=99.82 \mathrm{SD}=8.95)$ scored higher than males $(\mathrm{M}=94.32, \mathrm{SD}=9.11)$, and the effect size $(\mathrm{d}=.60)$ was large enough to indicate that the difference between these two groups was consistent enough to be really important.

The results provided further evidence for the findings of the study done by Harasty et al., (1997), who claimed that females are better in languageassociated activities due to the larger Wernicke and Broca cortical regions. The finding are also in line with Sabbatini (2000) who provided clear evidence for sex differences in the brain functional organization for language learning, indicating larger Wernicke and Broca areas in females' brain, Accordingly, since brain activation in females engages more diffuse neural systems which involves both right and left inferior frontal gyrus regions (Shaywitz et al., 1995), it seems logical to conclude that females shall outperform males in learning suprasegmental features due to the fact that these features tend to be processed in the language related regions of the left brain rather than the right hemisphere regions.

In addition to the above mentioned similar biological findings, the results of the study were compatible with those of many experimental studies, such as Brend (1975), in which it was found that women use intonation patterns differently and to a greater extent when compared to men; and Rao (2013) who found that females, contrary to males, can alter their rhythm to a great extent to resemble their interlocutor's response.

Research Question 3: Testing the Correlation between Attitude towards L1 (Persian) and learning English suprasegmental features.

The third question of the study was formulated to find out if there existed any significant correlation between EFL learners' attitude towards L1 as measured by the Attitude towards Persian Questionnaire, and their scores on Suprasegmental Features Learning Test. Pearson correlation was used to explore any probable correlation between EFL learners' attitude towards L1 and their scores on suprasegmental features test. However, before presenting the results of the Pearson correlation test, the descriptive statistics are shown in Table 6.

Table 6. Descriptive statistics for SLT scores and attitude towards L1

\begin{tabular}{llcc}
\hline \multicolumn{1}{c}{ Groups } & N & Mean & Std. Deviation \\
\hline SLT scores & 200 & 97.79 & 9.37 \\
Attitude towards L1 & 200 & 109.85 & 4.22 \\
\hline
\end{tabular}

As can be seen in Table 6, the mean score for shows the results of Pearson Product Moment SLT equals 97.97 and the mean scores for the attitude towards L1 is 109.85 . The following table

Correlation.

Table 7. Pearson correlation between attitude towards L1 and SLT scores

\begin{tabular}{ccc}
\hline & & SLT Scores \\
\hline Attitude towards & Pearson Correlation & .027 \\
L1 & Sig. (2-tailed) & .704 \\
& $\mathrm{~N}$ & 200 \\
\hline
\end{tabular}

As can be seen in Table 7, no statistically significant correlation was found between attitudes towards L1 and suprasegmental features learning (r $(198)=.02, \mathrm{p}>.05, \mathrm{~N}=200)$. Thus, since the null hypothesis was not rejected, we concluded that there was no significant correlation between the two specified variables.
To find the reason of such finding, 15 students, who gained high scores in suprasegmental features test and did the same in L1 questionnaire, were interviewed and asked to explain why they observed these features very well although they respected their mother tongue to a great extent. Most of the students reported their high motivation to learn 
English due to the fact that in today's world, having native like proficiency in English, helps people become more successful in both educational and business fields. Two of them reported that suprasegmental features, especially assimilation, are facilitative in understating native speakers and stated that they really enjoy speaking like them. In fact, students' declarations were directly related to their attitude. Not only did they not perceive English or any foreign languages as a threat to their identity and their mother tongue, but also considered it as a complementary factor to their success and improvement.

The results of the interview session were in line with that of Pullen (2011) who reported that advanced Turkish learners of English did not perceive a native-like pronunciation as a threat to their cultural identity. Moreover, it is wise to take this fact into account that learning each part of a language is not just a matter of attitude and many other factors including IQ level, way of teaching, emotional status of the learners and etc. can affect language learning process.

Research Question 4: Testing the correlation between attitude towards L2 (English) and learning suprasegmental features.

A Pearson product -moment correlation was computed to assess the relationship between attitude towards L2 (English) and learning suprasegmental features. Before presenting the correlation results, Table 8, presents the descriptive statistics.

Table 8. Descriptive statistics for SLT scores and attitude towards L2

\begin{tabular}{cccc}
\hline Groups & $\mathrm{N}$ & Mean & Std. Deviation \\
\hline SLT scores & 200 & 97.79 & 9.37 \\
Attitude towards L2 & 200 & 187.72 & 13.51 \\
\hline
\end{tabular}

As can be seen in Table 8, the mean for SLT scores is 97.97 and 187.72 for attitude towards L2.
The results of Pearson correlation can be seen in Table 9.

Table 9. Pearson correlation between attitude towards L2 and SLT scores

SLT Scores

\begin{tabular}{ccc}
\hline Attitude towards & Pearson Correlation & $.341^{* *}$ \\
L2 & Sig. (2-tailed) & .00 \\
& $\mathrm{~N}$ & 200 \\
\cline { 2 - 3 }
\end{tabular}

**.Correlation is significant at the 0.01 level (2-tailed)

As indicated in Table 9, a statistically significant positive correlation was found between attitudes towards L2 and learning suprasegmental features at the significant level of $0.01(\mathrm{r}(198)=.34$ $\mathrm{p}<.01, \mathrm{~N}=200)$. This means that as positive attitude towards L2 increases, the suprasegmental features learning ability will increase too. The strength of the relationship ( $\mathrm{R}$ squared value) is .116 , showing small effect size and that the two variables have $11.6 \%$ of common variance.

The findings are in line with the results of many other studies such as Moyer (2007), and Pullen (2011) who pointed out that foreign language learners with positive attitude towards second culture and language, tend to integrate with the target society and therefore adopt native like accent, and pronunciation (Pourhosein Gilakjani, 2012; Zhang \& Yin, 2009). The findings also supported the general concept of the effectiveness of attitude in language learning process as reported previously in many studies (Rukh, 2014; Martinsen \& Alvord, 2012). The results of the study further confirmed Elliot's (1995) findings, where positive attitude was found to be the main variable in relation to language pronunciation, implying that students who were more concerned about their pronunciation, had more positive attitude towards target language and; therefore, were more successful in imitating correct pronunciation. The same finding was also observed in Cenoz and Garcia Lecumberri (1999) who stated that the positive attitude towards a target language and its culture, leads the person to choose the pronunciation with which they are identified for the second language. Similar results were also reported by Moyer (2007), who confirmed that language attitudes specifically towards the target language are significant for acquiring native like accent, and that positive orientations are important for attaining authenticity in accent. To sum up, although no specific study has investigated the effect of attitude on learning intonation, assimilation and word stress, similar studies conducted on other elements of suprasegmental features, as mentioned above, have confirmed the effectiveness of positive attitude towards L2 on learning these features.

\section{CONCLUSION}

The importance of suprasegmental features, including intonation, word stress, assimilation, reduction, tone, and etc., in language learning has been highlighted by different researchers (Ladefoged, 2010; Roach, 2002), and previous 
research findings have confirmed the effective role of the above mentioned variables in learning language and specifically learning pronunciation (Anaki et al., 1998; Ferrand \& Bloom, 1996; Gardner, 1976).

The present study aimed at finding the possible correlation between non-biological factors such as attitudes towards L1 and L2, on the one hand, and biological factors such as brain hemispheric dominance and gender and learning suprasegmental features, on the other hand. The results of statistical analyses revealed that brain hemispheric dominance and gender affect learning suprasegmental features to a great extent. A statistically significant relationship was also found between attitudes towards L2 and learning these features. However, no significant relationship was observed between attitude towards L1 and learning suprasegmental features of L2.

Undoubtedly, the findings of the present smallscale research project are not adequate to generalize the results to other contexts. However, theoretical implication of this study is of great importance. Due to the fact that there are still controversial debates regarding laterality of intonation and suprasegmental features, the finding of this study can provide support for the left hemisphere superiority for linguistic intonation and other suprasegmental features implying that, the more linguistic the acoustic signal and its content, the more left hemisphere regions are engaged.

In addition to theoretical implications, there are some pedagogical issues to be discussed. Since suprasegmental features are related to pronunciation, and since pronunciation is of great importance in both listening and speaking skills, the language instructors are recommended to use different methods and facilities such as visual aids to activate the learners' left hemispheres. In other words, since all of the functions of each hemisphere are not equally empowered, the instructors can take benefit from other functions of the left hemisphere to strengthen suprasegmental features learning. Due to the fact that left hemisphere has a great effect on learning suprasegmental features,language teachers should take benefit from the activities which involve both hemispheres, especially the left hemisphere, in listening and speaking task in which pronunciation and suprasegmental features have crucial roles.

Regarding listening tests, assessors, especially those who assess beginner language learners or children, should take this fact into account that learning suprasegmental features is not only a matter of being assiduous learner, but also a matter of many biological and non-biological factors. Thus, they should avoid making decisions solely based on the performance of the students and should consider other factors such as affective and environmental factors in their final assessment and decisions, since in many cases, teachers or assessors' educational decisions can change the learning process of a language learner.

On the other hand, since attitude towards L2 has positive relationship with learning suprasegmental features, the instructors and materials developers should pay attention to the way they present L2 culture and language. Since one of the main sources of information about the target culture are the instructors, they should provide the learners with interesting and authentic information about the second or foreign language context and culture and help them have positive attitudes towards the target situation and language. In the same way, the authors of the foreign languages books should try to present fabulous aspects of the target society and language and avoid focusing on negative aspects of second or foreign language or culture in their textbooks, and other types of materials. Visual presentation of interesting aspects of the target language and culture, for example, can attract learners easily and motivate L2 learners to try to acquire native like pronunciation and accent.

\section{REFERENCES}

Anaki, D., Faust, M., \& Kravetz, S. (1998). Cerebral hemispheric asymmetries in processing lexical metaphors. Neuropsychologia, 36(4), 353-362. Archives of Neurology, 38(12), 742-749.

Arzouan, Y., Goldstein, A., \& Faust, M. (2007). Dynamics of hemispheric activity during metaphor comprehension: Electrophysiological measures. Neuroimage, 36(1), 222-231.

Baum, S., \& Pell, M. (1999). The neural bases of prosody: Insights from lesion studies and neuroimaging. Aphasiology, 13(8), 581-608.

Behrens, S. (1989). Characterizing sentence intonation in a right hemisphere-damaged population. Brain and Language, 37(2), 181200.

Blumstein, S., \& Cooper, W. (1974). Hemispheric processing of intonation contours. Cortex, 10(2), 146-158.

Brazil, D., Coulthard, M., \& Johns, C. (1980). Discourse intonation and language teaching. London: Longman.

Brend, R. (1975). Male-female intonation patterns in American English. In B. Thorne \& N. Henly (Eds.), Language and sex: Difference and dominance (pp. 84-87). Rowley: MA: Newbury House.

Broadbent, D., \& Gregory, M. (1964). Accuracy of recognition for speech presented to the right and left ears. Quarterly Journal of Experimental Psychology, 16(4), 359-360.

Broca, P. (1861). Remarks on the seat of the faculty of articulated language, following an observation of aphemia (loss of speech). Bulletin De La Society Anatomique, 6, 330357. 
Bryden, M. (1963). Ear preference in auditory perception. Journal of Experimental Psychology, 65(1), 103-105.

Cakir, I., \& Baytar, B. (2014). Foreign language learners' views on the importance of learning the target language pronunciation. Journal of Language and Linguistic Studies, 10(1), 99110. Retrieved from http://jlls.org.

Cenoz, J., \& Garcia Lecumberri, M. (1999). The acquisition of English pronunciation: learners' views. International Journal of Applied Linguistics, 9(1), 3-15.

Chun, D. (2002). Discourse intonation in L2. Amsterdam: John Benjamins.

Corkin, S. (1978). The role of different cerebral structures in somesthetic perception. Handbook of perception, 6, 105-155.

Darabad, A. (2003). Attitude towards foreign language, corrective feedback, and oral accuracy. International Journal of Linguistics, 5(2), 116-134.

DavariArdekani, N., \& Jalilian, A. (2013). Sociolinguistics: Language planning, and language attitudes. Tehran: Jame'eShenasan.

Davidoff, J. (1982). Studies with non-verbal stimuli. In J. G. Beaumont (Eds.), Divided visual field studies of cerebral organization (pp. 2955).London; Academic Press.

Devinsky, O., \& D'Esposito, M. (2004). Executive function and the frontal lobes. In $O$. Devinsky \&. M. D'Esposito (Eds.), Neurology of cognitive and behavioral disorders (pp. 302329).New York: Oxford University Press.

Edwards, L. (2007). Solutions placement test. Oxford: Oxford University Press.

Elliot, A. (1995). Field independence/dependence, hemispheric specialization, and attitude in relation to pronunciation accuracy in Spanish as a foreign language. The Modern Language Journal, 79(3), 356-371.

Eng, N., Obler, L., Harris, K., \& Abramson, A. (1996). Tone perception deficits in Chinesespeaking Broca's aphasics.Aphasiology, 10(6), 649-656.

Ferrand, C., \& Bloom, R. (1996). Gender differences in children's intonational patterns. Journal of Voice, 10(3), 284-291.

Fotovatnia, Z., \&Omidi, M. (2013). The effect of exposure to the visual medium on learning pronunciation and word stress of L2 learners. TPLS, 3(5), 769-775.

Friederici, A., \& Alter, K. (2004). Lateralization of auditory language functions: A dynamic dual pathway model. Brain and Language, 89(2), 267-276.

Gandour, J., \& Dardarananda, R. (1983). Identification of tonal contrasts in Thai aphasic patients. Brain and Language, 18(1), 98-114.

Gandour, J., Tong, Y., Wong, D., Talavage, T., Dzemidzic, M., \&Xu, Y. et al. (2004).
Hemispheric roles in the perception of speech prosody. Neuroimage, 23(1), 344-357.

Gardner, R. (1976). Attitude and motivation in second language learning: course related changes. Canadian Modern Language Review, 32(3), 243-266.

Geschwind, N. (1975). The apraxias: Neural mechanisms of disorders of learned movement: The anatomical organization of the language areas and motor systems of the human brain clarifies apraxic disorders and throws new light on cerebral dominance. American Scientist, 63, 188-195.

Goldstein, M., Joynt, R., \& Hartley, R. (1975). The Long-Term Effects of Callosal Sectioning. Arch Neurol, 32(1), 52-56. doi:10.1001/archneur.1975.00490430074014.

Goodglass, H., \& Calderon, M. (1977). Parallel processing of verbal and musical stimuli in right and left hemispheres. Neuropsychologia, 15(3), 397-407.

Harasty, J., Double, K., Halliday, G., Kril, J., \& McRitchie, D. (1997). language-associated cortical regions are proportionally larger in the female brain. Archives of Neurology, 54(2), 171-176.

Jäncke, L., Wüstenberg, T., Scheich, H., \&Heinze, H. (2002). Phonetic perception and the temporal cortex. Neuroimage, 15(4), 733-746.

Jenkins, J. (2004). Research in teaching pronunciation and intonation. Annual Review of Applied Linguistics, 24, 109-125.

Jones, W. (1950). Attitude towards Welsh as a second language. British Journal of Educational Psychology, 20(2), 117-132.

Jusczyk, P. (1997). The role of infant speech perception capacities in discovering the sound structure of the native language. Journal of Acoustic Society of America, 101(5), 31-92.

Kansaku, K., Yamaura, A., \& Kitazawa, S. (2000). Sex differences in lateralization revealed in the posterior language areas. Cerebral Cortex, 10(9), 866-872.

Kimura, D. (2000). Sex and cognition (pp. 31-59). Cambridge, Mass.: MIT Press.

Klein, D., Zatorre, R., Milner, B., \& Zhao, V. (2001).A Cross-linguistic PET study of tone perception in Mandarin Chinese and English speakers. Neuroimage, 13(4), 646-653.

Ladefoged, P. (2010). A course in phonetics. Fort Worth: Harcourt Brace Jovanovich College Publishers.

Lancker, V., \& Fromkin, V. (1973).Hemispheric specialization for pitch and" tone": Evidence from Thai. Journal of Phonetics, 1, 101-109.

Martinsen, R., \& Alvord, S. (2012). On the relationship between L2 pronunciation and culture. Spanish in Context, 9(3), 443-465.

Mateer, C. (1983). Motor and perceptual function of the left hemisphere and their interaction. In S. 
Segalowitz (Eds.), Language functions and brain organization (pp. 145-170). New York: Academic Press.

McKeever, W., \& Jackson, T. (1979). Cerebral dominance assessed by object- and colornaming latencies: Sex and familial sinistrality effects. Brain and Language, 7(2), 175-190.

Meyer, V., \& Yates, A. (1955). Intellectual changes following temporal lobotomy for psychomotor epilepsy: preliminary communication. Journal of Neurology, Neurosurgery \& Psychiatry, 18(1), 44-52.

Milner, B. (1968). Visual recognition and recall after right temporal-lobe excision in man. Neuropsychologia, 6(3), 191-209.

Moen, I. (1993). Phonological deviations in Norwegian conduction aphasia: Testing a Model of Non-linear phonology. Nordic Journal of Linguistics, 16(02), 99-103

Moir, A., \& Jessel, D. (1989). Brain sex: the real difference between men and women. New York: Dell Publishing.

Moyer, A. (2007). Do language attitudes determine accent? A study of bilinguals in the USA. Journal of Multilingual and Multicultural Development, 28(6), 502-518. doi:10.2167/jmmd514.0

Nash, R. (1971). Phonemic and prosodic interference and their effects on intelligibility. In A. Rigault \& $R$. Charbonneau (Eds.), Proceedings of the 7th International Congress of Phonetic Sciences, (pp. 570-573). The Hague: Mouton

Pachler, N., \& Field, K. (1997). Learning to teach modern foreign languages in the secondary school. London: Routledge.

Pell, M. (1999). Fundamental frequency encoding of linguistic and emotional prosody by right hemisphere damaged speakers. Brain and Language, 69, 161-192.

Pourhosein Gilakjani, A. (2012). A study of factors affecting EFL learners' English pronunciation learning and the strategies for instruction. International Journal of Humanities and Social Science, 2(3), 119-128.

Pullen, E. (2011). Cultural identity, pronunciation, and attitudes of Turkish speakers of English: Language identity in an EFL context. In. J. Levis \& K. LeVelle (Eds.). Proceedings of the $3^{\text {rd }}$. Pronunciation in Second Language Learning and Teaching Conference, Sept. 2011. (pp. 65-83). Ames, IA: Iowa State University.

Ransil, B., \& Schachter, S. (1994). Test-retest reliability of the Edinburgh Handedness Inventory and global handedness preference measurements, and their correlation. Perceptual and Motor Skills, 79(3), 13551372.

Rao, G. (2013). Measuring phonetic convergence: segmental and suprasegmental speech adaptations during native and non-native talker interactions (PhD Dissertation). The University of Texas at Austin.

Rapp, A., Leube, D., Kircher, M., Erb, W., \& Grodd, T. (2004). Neural correlates of metaphor processing. Cognitive Brain Research, 20(3), 395-402.

Ratcliff, G. (1982). Disturbances of spatial orientation associated with cerebral lesions. InM. Potegal (Eds.), Spatial abilities: development and physiological foundations (pp. 301-331), New York: Academic Press.

Roach, P. (2002).English phonetics and phonology. Cambridge [Cambridge shire]: Cambridge University Press.

Rogerson-Revell, P., \& Miller, L. (1994).Developing pronunciation skill through self-assess learning. In D.Gardner \& L. Miller, Directions in self-assess language learning (1st Ed.). Hong Kong: Hong Kong University Press.

Rukh, S. (2014). Students' attitude towards English language learning and academic achievement: A case of business students in Punjab. European Academic Research, 2(4), 55695612.

Sabbatini, R. (2000). Are there differences between the brains of males and females?

Cerebromente.org.br. Retrieved 14 April 2014, from

http://www.cerebromente.org.br/n11/mente/eis ntein/cerebro-homens.html

Schirmer, A., Alter, K., Kotz, S., \& Friederici, A. (2001). Lateralization of prosody during language production: A lesion Study. Brain and Language, 76(1), 1-17.

Shankweiler, D., \& Studdert-Kennedy, M. (1967). Identification of consonants and vowels presented to left and right ears. Quarterly Journal of Experimental Psychology, 19(1), 59-63.

Shaywitz, B., Pugh, K., Todd Constable, R., Shaywitz, S., Bronen, R., \& Fulbright, R. et al. (1994). Localization of semantic processing using functional magnetic resonance imaging. Human Brain Mapping, 2(3), 149-158.

Shaywitz, B., Shaywltz, S., Pugh, K., Constable, R., Skudlarski, P., \& Fulbright, R. et al. (1995). Sex differences in the functional organization of the brain for language.Nature, 373, 607-609.

Shipley-Brown, F., Dingwall, W., Berlin, C., YeniKomshian, G., \& Gordon-Salant, S. (1988). Hemispheric processing of affective and linguistic intonation contours in normal participants. Brain and Language, 33(1), 1626.

Soars, L., \& Soars, J. (2009). New headway English course (4th Ed.). Oxford: Oxford University Press. 
Sparks, R., \& Ganschow, L. (1991). Foreign language learning differences: affective or native language aptitude differences? The Modern Language Journal, 75(1), 3-16.

Stringaris, A., Medford, N., Giampietro, V., Brammer, M., \& David, A. (2007). Deriving meaning: Distinct neural mechanisms for metaphoric, literal, and non-meaningful sentences. Brain and Language, 100(2), 150162.

Suter, R. (1976). Predictors of pronunciation accuracy in second language learning. Language Learning, 26(2), 233-253.

Tucker, D., Watson, R., \& Heilman, K. (1977). Discrimination and evocation of affectively intoned speech in patients with right parietal disease. Neurology, 27(10), 947-947.

Wang, Y., Jongman, A., \& Sereno, J. (2001). Dichotic perception of Mandarin tones by Chinese and American listeners. Brain and Language, 78(3), 332-348.
Weintraub, S., Mesulam, M.M., Kramer, L. (1981). Disturbances in Prosody. A right-hemisphere contribution to language. Arch Neurol. 38(12):742-744.

Yin, R. (1970). Face recognition by brain-injured patients: A dissociable ability? Neuropsychologia, 8(4), 395-402

Yiu, E., \& Fok, A. (1995). Lexical tone disruption in Cantonese aphasic speakers. Clinical Linguistics \& Phonetics, 9(1), 79-92.

ZainolAbidin, M., Pour-Mohammadi, M., \& Alzwari, H. (2012). EFL students' attitudes towards learning English language: the case of Libyan secondary school students. Asian Social Science, 8(2).119-134.

Zhang, F., \& Yin, P. (2009). A study of pronunciation problems of English learners in China. Asian Social Science, 5(6).141-145.

Zurif, E. (1974). Auditory lateralization: Prosodic and syntactic factors. Brain and Language, 1(4), 391-404. 